\title{
Guest Editorial: Special Issue on Managing and Mining Massive Graphs
}

\author{
Haixun Wang ${ }^{1} \cdot$ Jeffrey Xu Yu${ }^{2}$
}

Published online: 19 June 2015

(C) Springer Science+Business Media New York 2015

Many applications need to handle very large graphs, including the web graph, social network graph, knowledge graphs, biology graphs, etc. We are facing challenges at all levels from system infrastructures to programming models for managing and analyzing large graphs. Unlike other types of big data, graphs are highly interconnected, which enables graph to represent complex data structures in a variety of applications, but at the same time, also makes graph query processing and graph analytics extremely difficult, especially when the graph is big. Much effort has been devoted to managing and mining massive graphs. These work include graph systems, graph query languages, graph access methods, basic operators such as graph reachability and shortest distance queries, and advanced analytics on graphs. There is also a big push from the application side. Linked data, social networks, customer relationship management, biological and chemical applications are all pushing for breakthroughs in this area.

This special issue presents high quality research ideas related to the graph data management and mining area.

The first paper of this issue: The $G^{*}$ Graph Database: Efficient Managing Large Distributed Dynamic Graphs by Alan G. Labouseur, Jeremy Birnbaum, Paul W. Olsen Jr., Sean R. Spillane, Jayadevan Vijayan, Jeong-Hyon Hwang, and Wook-Shin Han presents a graph database system $\mathrm{G}^{*}$ to process complex queries over large graphs in parallel with the features of sharing computation across graphs.

\footnotetext{
$凶 \quad$ Jeffrey Xu Yu

yu@se.cuhk.edu.hk

Haixun Wang

haixun@google.com

1 Google Research, Mountain View, CA, USA

2 The Chinese University of Hong Kong, Hong Kong, China
} 
The second paper of this issue: Analysis of Schema Structures in the Linked Open Data Graph based on Unique Subject URIs, Pay-level Domains, and Vocabulary Usage by Ansgar Scherp Malte Knauf Thomas Gottron studies a fundamental issue of finding matches of a given query graph over a large data graph, and proposes a new techniques to find approximate matches by combining/aligning paths specified by the user-given graph query.

The third paper of this issue: Approximate Querying of RDF Graphs via Path Alignment by Roberto De Virgilio Antonio Maccioni Riccardo Torlone addresses the schema structures in the Linked Open Data (LOD) graph which is a graph representing knowledge with various of types defined by major industries, academia, governmental institutes, etc.

The fourth paper of this issue: Intra Graph Clustering using Collaborative Similarity Measure by Waqas Nawaz, Kifayat-Ullah Khan, Young-Koo Lee, and Sungyoung Lee presents a new collaborative similarity measure to cluster vertices in a large graph based on their similarity based on topological structures and attributes.

We would like to thank all those who contributed to this special issue, Divy Agrawal, and Amit P. Sheth, the editor-inchiefs, for their cooperation, the reviewers for their thorough comments that help in enhancing the quality of the papers, and the authors for submitting their papers to this special issue. 